\title{
Monozygotic female twins discordant for Silver-Russell syndrome and hypomethylation of the H19-DMR
}

\author{
Kazuki Yamazawa $\cdot$ Masayo Kagami · \\ Maki Fukami · Keiko Matsubara · Tsutomu Ogata
}

Received: 14 April 2008/Accepted: 14 July 2008/Published online: 16 August 2008

(C) The Japan Society of Human Genetics and Springer 2008

\begin{abstract}
Silver-Russell syndrome (SRS) is characterized by growth failure and dysmorphic features, and is frequently caused by hypomethylation of the paternally derived H19-DMR (epimutation). We observed 5 8/12year-old female twins discordant for SRS. One twin exhibited SRS-compatible features, such as pre- and postnatal growth failure, relative macrocephaly, triangular face, left hemihypotrophy, and bilateral fifth finger clinodactyly, whereas the other twin showed apparently normal phenotype. Microsatellite analysis for 26 loci on multiple chromosomes showed monozygosity. Methylation analysis for the H19-DMR indicated epimutation in roughly half of cells in the affected twin and normal patterns in the unaffected twin and the parents. X-inactivation analysis revealed random $\mathrm{X}$-inactivation with a nearly identical pattern between the twins. The discordant methylation pattern of the H19-DMR may primarily be due to a failure to maintain the DNA methyltransferase-1-dependent methylation imprint around the pre-implantation S phase, because such failure would result in the production of two different cell clones, one with normally methylated DMR and the other with demethylated DMR, leading to the separation of cells with different characters and resultant twinning.
\end{abstract}

K. Yamazawa ( $₫) \cdot$ M. Kagami · M. Fukami - K. Matsubara · T. Ogata

Department of Endocrinology and Metabolism,

National Research Institute for Child Health and Development,

Tokyo 157-8535, Japan

e-mail: kyamazawa@nch.go.jp

K. Yamazawa

Department of Pediatrics, Keio University School of Medicine,

Tokyo 160-8582, Japan
Keywords Silver-Russell syndrome . Monozygotic twin · Discordance - Methylation · H19-DMR $\cdot$ X-inactivation $\cdot$ DNMT1

\section{Introduction}

Silver-Russell syndrome (SRS; MIN 180860) is a developmental disorder characterized by pre- and postnatal growth failure, relative macrocephaly, triangular face, hemihypotrophy, and fifth-finger clinodactyly (Hitchins et al. 2001). Recent studies have shown that hypomethylation (epimutation) of the paternally derived differentially methylated region in the upstream of $H 19$ (H19-DMR) on chromosome 11p15 accounts for 30-65\% of SRS patients (Gicquel et al. 2005; Bliek et al. 2006; Eggermann et al. 2006; Netchine et al. 2007). In this regard, it is known that a common enhancer is shared by the paternally expressed gene IGF2 (insulin-like growth factor 2) and the maternally expressed gene $H 19$ (Leighton et al. 1995b), and that the enhancer exerts its effects on IGF2 or $H 19$ depending on the methylation status of the H19-DMR (Leighton et al. 1995a; Thorvaldsen et al. 1998). This alternative enhancer effect is mediated by an insulator protein CTCF that binds to the unmethylated H19-DMR of maternal origin, but not to the methylated H19-DMR of paternal origin. Indeed, seven CTCF binding sites have been identified within the H19-DMR (Bell and Felsenfeld 2000; Hark et al. 2000). Thus, it has been postulated that the hypomethylation of the paternally derived $H 19$-DMR results in maternalization of the IGF2-H19 imprinted domain, leading to the development of SRS because of reduced IGF2 expression (Gicquel et al. 2005). Although the exaggerated H19 expression might also exert some clinical effect, this possibility is unlikely because the primary function of $\mathrm{Hl}$ in 
the bodies appears to be tumor suppression (Hao et al. 1993; Juan et al. 2000). This finding, together with the identification of maternal uniparental disomy for chromosome 7 [upd(7)mat] in 7-10\% of SRS patients (Hitchins et al. 2001), implies that SRS primarily represents an epigenetic disorder.

Notably, five monozygotic twin pairs discordant for SRS have been reported (Nyhan and Sakati 1977; Samn et al. 1990; Bailey et al. 1995; Sagot et al. 1996; Gicquel et al. 2005). In addition, the H19-DMR analysis has been performed in a single female twin pair, identifying a discordant methylation pattern (Gicquel et al. 2005). Here, we report monozygotic twins discordant for SRS and hypomethylation of the H19-DMR, and discuss underlying factors for such discordance.

\section{Materials and methods}

\section{Case report}

This Japanese female patient and her twin sister were conceived naturally to an unrelated and healthy 52-year-old father and 37-year-old mother. They were found to be monochorionic diamniotic twins by ultrasonographic studies at the first trimester. Because of the advanced maternal age, amniocentesis was carried out at 15 weeks of gestation, showing a 46,XX karyotype in both of the twins. The patient exhibited growth retardation since 20 weeks of gestation, while the twin sister grew well. Since the patient frequently showed decelerated fetal heart rate at 28 weeks of gestation, the twins were delivered by an emergent cesarean section.

At birth, the patient was $25.0 \mathrm{~cm}$ in length, $494 \mathrm{~g}$ in weight, and $24.0 \mathrm{~cm}$ in occipitofrontal head circumference (OFC), whereas the twin sister was $32.0 \mathrm{~cm}$ in length, $734 \mathrm{~g}$ in weight, and $24.5 \mathrm{~cm}$ in OFC (no reference growth data in neonates born at 28 weeks). Both twins were admitted to the neonatal intensive care unit and received respiratory and nutrition management for long terms (10 months for the patient and 5 months for the sister). The placenta of $280 \mathrm{~g}$ was shared by the twin, but there was no discernible interconnecting vascular anastomosis. At 2 years of age, the patient was diagnosed as having SRS on the basis of a constellation of clinical features that met the diagnostic criteria for SRS (Hitchins et al. 2001), such as pre- and postnatal growth failure, triangular face, relative macrocephaly, left hemihypotrophy, and bilateral fifth finger clinodactyly. Her psychomotor development appeared to be age-appropriate. Endocrine studies for short stature were normal, as were radiological studies. By contrast, the twin sister showed catch-up growth and manifested no SRS-like features. At 5 8/12 years of age, the patient measured $91.2 \mathrm{~cm}(-4.5 \mathrm{SD})$, weighed $12.8 \mathrm{~kg}$ $(-2.1 \mathrm{SD})$, and had an OFC of $48.3 \mathrm{~cm}(-1.4 \mathrm{SD})$, whereas the twin sister measured $102.0 \mathrm{~cm}(-1.9 \mathrm{SD})$, weighed $13.6 \mathrm{~kg}(-1.9 \mathrm{SD})$, and had an OFC of $49.1 \mathrm{~cm}$ $(-0.9 \mathrm{SD})$.

Microsatellite analysis

This study was approved by the Institutional Review Board Committee at the National Center for Child health and Development, and written informed consent was obtained from the parents. A total of 26 loci on multiple chromosomes were genotyped, using leukocyte genomic DNA. In brief, a segment encompassing each locus was PCRamplified with a fluorescently labeled forward primer and an unlabeled reverse primer, and was determined for the physical size on an ABI PRISM 310 autosequencer using GeneScan software (Applied Biosystems, Foster City, CA).

Methylation analysis

Combined bisulfite restriction analysis (COBRA) and bisulfite sequencing were performed for two regions within the H19-DMR, i.e., a 317-bp region distal to the CTCF binding sites (DMR-A) (Vu et al. 2000) and a 435-bp region encompassing the CTCF binding site 6 (DMR-B), using leukocyte genomic DNA (Fig. 1a). The DMR-A and DMR-B were PCR-amplified with primers that hybridize to both methylated and unmethylated alleles because of absent $\mathrm{CpG}$ dinucleotides within the primer sequences.

For COBRA, the PCR products were digested with methylated allele-specific restriction enzymes (BsaBI and MwoI for the DMR-A; AfIII and Hpy188I for the DMR-B) (Fig. 1a). Subsequently, the methylation index was obtained using peak heights of digested and undigested fragments on the 2100 Bioanalyzer (Agilent, Santa Clara, CA). To define the normal range for the methylation indices, 40 control subjects were similarly studied.

For bisulfite sequencing, the PCR products were subcloned with TOPO TA Cloning Kit (Invitrogen, Carlsbad, CA), and multiple clones were subjected to direct sequencing on the CEQ 8000 autosequencer (Beckman Coulter, Fullerton, CA). The SNPs within the DMR-A (rs2251375) and the DMR-B (rs11564736, rs 10732516, $r s 34610866, r s 2071094, r s 35678657$, and $r s 2107425)$ were also genotyped, to identify the parental origin of each clone.

$\mathrm{X}$-inactivation analysis

$\mathrm{X}$-inactivation pattern was examined by the previously reported methods (Muroya et al. 1999). In brief, leukocyte genomic DNA was PCR-amplified with a 

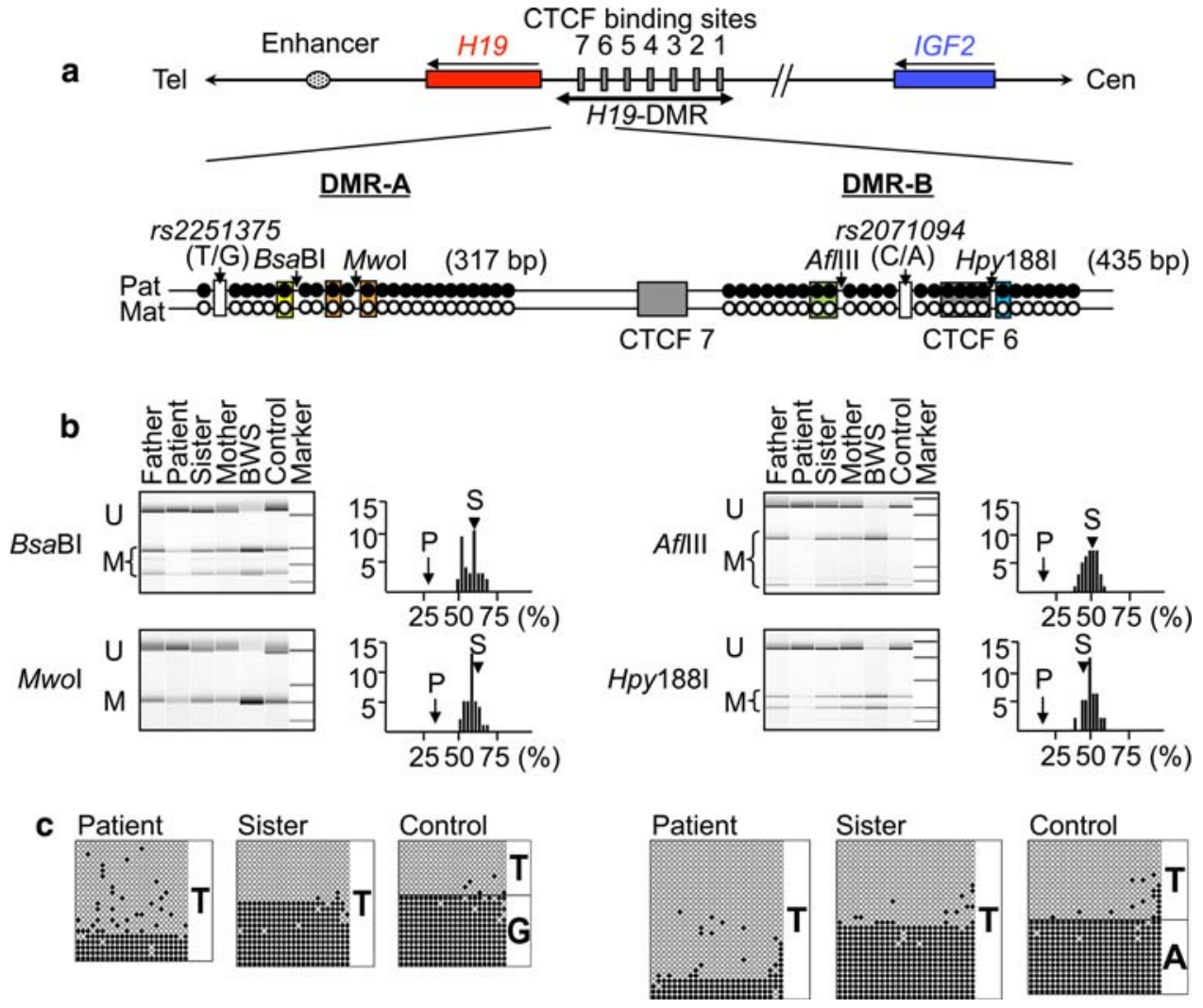

Fig. 1 Methylation analysis of the H19-DMR. a The regional physical map of the IGF2-H19 imprinted domain and the location and the structure of the DMR-A and the DMR-B examined in this study. The H19-DMR resides in the upstream of $H 19$ and contains seven CTCF binding sites (shown in gray boxes 1-7). A common enhancer (shown with a stippled ellipse) is shared by the paternally expressed gene IGF2 (shown in blue) and the maternally expressed gene $H 19$ (shown in red), and the enhancer exerts its effects on IGF2 when the H19-DMR is methylated after paternal transmission (filled circles) and on $H 19$ when the H19-DMR is unmethylated after maternal transmission (open circles). The DMR-A (317 bp) resides distal to the CTCF binding sites and harbors $23 \mathrm{CpG}$ dinucleotides, methylated allele-specific $B s a \mathrm{BI}$ and $M w o \mathrm{I}$ restriction sites, and the T/ G SNP (rs2251375) (depicted with a white box). After bisulfite treatment, this region is digested with $B s a \mathrm{BI}$ when the cytosine at the 6th $\mathrm{CpG}$ dinucleotide (indicated with a yellow rectangle) is methylated and with MwoI when the two cytosines at the 9th and the 11th $\mathrm{CpG}$ dinucleotides (indicated with two orange rectangles) are methylated. The primer sequences used were: $5^{\prime}$-AACCCCTTCCTACCACCATC- $3^{\prime}$ and $5^{\prime}$-GGGTTTGGGAGAGTTTGTGA- ${ }^{\prime}$. The DMR-B (435 bp) encompasses the CTCF binding site 6 (shown with a gray box) and contains $26 \mathrm{CpG}$ dinucleotides, methylated allelespecific AflIII and Hpy188I restriction sites, and the C/A SNP (rs2071094) (depicted with a white box). After bisulfite treatment, this region is digested with AfIIII when the cytosines at the eighth and the ninth $\mathrm{CpG}$ dinucleotides (indicated with green rectangles) are methylated and with Hpy188I when the cytosine at the 20th $\mathrm{CpG}$

fluorescently labeled forward primer and an unlabeled reverse primer flanking the highly polymorphic CAG repeat region and the two methylation sensitive HpaII sites at exon 1 of $A R$, before and after HpaII digestion

dinucleotide (indicated with a blue rectangle) is methylated. The primer sequences used were: 5'-TGGGAGGAGATATTAGGGGAATA- $3^{\prime}$ and $5^{\prime}$-TCCCAAACCATAACACTAAAACC- $3^{\prime}$. b The results of the COBRA for the DMR-A (left) and the DMR-B (right). The actual electrophoresis images indicate that, for all the $B s a \mathrm{BI}$ and the MwoI sites in the DMR-A and the AfIIII and the Hpy188I sites in the DMR-B, both unmethylated clone-specific bands $(U)$ and methylated clone-specific bands $(M)$ are found in the father, the sister, and the mother as well as in a control subject, whereas $U$ is predominant in the patient and $M$ is predominant in the BeckwithWiedemann syndrome (BWS) patient with upd(11p15)pat. As compared with the methylation indices of the normal control subjects depicted as histograms (the horizontal axis the methylation index with an interval of $2.5 \%$; the vertical axis the subject number), the methylation indices of the patient $(P)$ (shown with arrows) are below the normal range, and those of the twin sister $(S)$ (shown with arrowheads) remain within the normal range. c Bisulfite sequencing results of the DMR-A (left) and the DMR-B (right) in the patient, the sister, and a control subject. Each line indicates a single clone, and each circle denotes a $\mathrm{CpG}$ dinucleotide; filled and open circles represent methylated and unmethylated cytosines, respectively. The T/G SNP ( $r$ 2251375) typing data within the DMR-A are indicated, as are the C/A SNP (rs2071094) typing data within the DMR-B (the $r s 2071094 \mathrm{C}$ allele has been converted into $\mathrm{T}$ allele after bisulfite treatment). The remaining five SNPs within the DMR-B were present in a homozygous status

(Fig. 2). The $\mathrm{X}$-inactivation ratio was calculated using the area under curve after compensation for unequal amplification of the two alleles caused by the difference in the product size. 


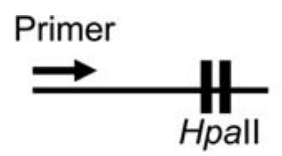

(CAG)n

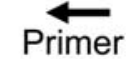

Patient
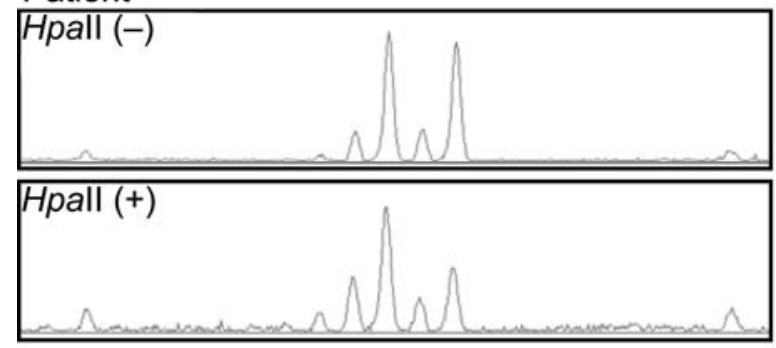

Sister
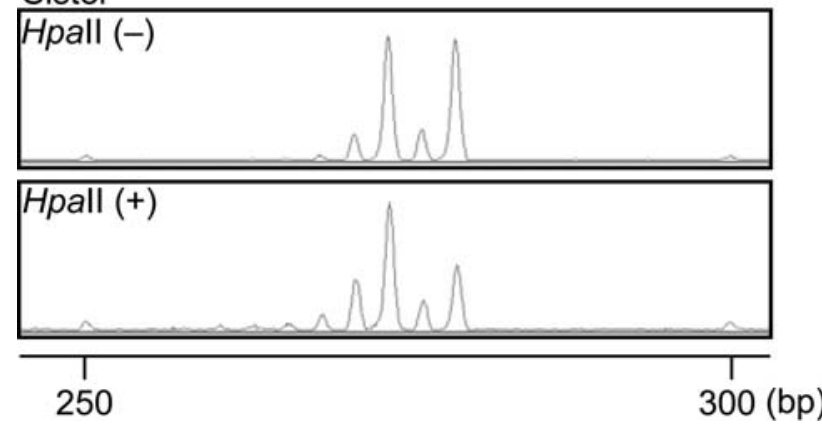

Fig. 2 X-inactivation analysis. The examined region contains a highly polymorphic CAG repeat region and two methylation sensitive HpaII sites. Thus, PCR products are obtained from both active and inactive $\mathrm{X}$ chromosomes before HpaII digestion and from inactive $\mathrm{X}$ chromosomes alone after HpaII digestion. The comparison of the area under the curves between two heterozygous peaks (273 and $279 \mathrm{bp}$ ) before and after HpaII digestion indicates that the two X chromosomes undergo random $\mathrm{X}$-inactivation with nearly identical patterns between the twins. The small peaks are by-products caused by slippage phenomenon

\section{Results}

Microsatellite analysis

All the genotyping results were identical between the twins, indicating monozygosity (Table 1). Furthermore, upd(7)mat was excluded, as was upd(11p15)mat involving the H19-DMR.

\section{Methylation analysis}

The methylation indices of the patient (the DMR-A, 28.0\% for the $B s a \mathrm{BI}$ site and $30.4 \%$ for the MwoI site; the DMRB, $17.4 \%$ for the AfIIII site and $17.1 \%$ for the Hpy $188 \mathrm{I}$ site) were below the normal ranges (the DMR-A, 48.1-64.7\% for the $B s a \mathrm{BI}$ site and $50.1-67.6 \%$ for the $M w o I$ site; the DMR-B, 39.8-59.8\% for the AfIIII site and 39.3-58.0\% for the Hpy188I site), whereas those of the twin sister, the father, and the mother were within the normal range (the DMR-A, $61.3,61.7$, and $62.3 \%$ for the $B s a \mathrm{BI}$ site and 64.6, 64.9, and $61.0 \%$ for the MwoI site; the DMR-B, 52.6, 52.7, and $51.5 \%$ for the $A f I I I$ site and $49.5,50.4$, and $52.9 \%$ for the Hpy188I site) (Fig. 1b). Furthermore, bisulfite sequencing revealed that the DMR-A and the DMR-B were predominantly hypomethylated in the patient and differentially methylated in the sister, although the SNP typing data for the seven SNPs were not informative for the parental origin of each clone in the twins (Fig. 1c). In the control subject, heterozygosity was detected for the T/G SNP (rs2251375) within the DMR-A and the C/A SNP ( $r$ 2071094) within the DMR-B, while the remaining five SNPs in the DMR-B were present in a homozygous status.

$\mathrm{X}$-inactivation analysis

Both the patient and the twin sister had random X-inactivation with a nearly identical pattern $(64 \%: 36 \%$ for the patient and 65\%:35\% for the sister) (Fig. 2).

\section{Discussion}

We observed monozygotic twins discordant for SRS and identified hypomethylation of the H19-DMR (epimutation) in the affected twin only. In this regard, the clinical diagnosis of SRS in the affected twin was based on the criteria proposed by Hitchins et al. (2001), and the epimutation was clearly demonstrated for the two regions within the H19DMR (DMR-A and DMR-B). Thus, although the difference in the clinical course might have some influence on the clinical and molecular discordance between the twins, the results imply that SRS in the affected twin is primarily caused by the epimutation occurring in the early fetal life.

The discordant methylation pattern of the H19-DMR may primarily be due to a failure to maintain the DNA methyltransferase-1 (DNMT1)-dependent methylation imprint around the pre-implantation $\mathrm{S}$ phase (Hirasawa et al. 2008). This notion has basically been suggested for monozygotic twins discordant for Beckwith-Wiedemann syndrome (BWS; MIM 130650) and hypomethylation of the KvDMR1 (Gaston et al. 2001; Weksberg et al. 2002). Indeed, such a failure would result in a hemimethylated daughter duplex that would subsequently be converted to a fully methylated and a fully unmethylated sister chromatid in the next S phase (Weksberg et al. 2002). This event would yield two different cell clones, one with normally methylated DMR with normal growth potential and the other with demethylated DMR with decreased growth potential, thereby leading to the separation of cells with different characters and resultant twinning discordant for 
Table 1 The results of microsatellite analysis

\begin{tabular}{|c|c|c|c|c|c|c|}
\hline Locus & Position & Mother & Patient & Sister & Father & Assessment \\
\hline D3S1314 & $3 q 28$ & 139 & $139 / 145$ & $139 / 145$ & $141 / 145$ & Biparental \\
\hline D7S2846 & $7 \mathrm{p} 14$ & 177 & $177 / 181$ & $177 / 181$ & 181 & Biparental \\
\hline D7S519 & $7 \mathrm{p} 13$ & $261 / 263$ & $261 / 263$ & $261 / 263$ & $261 / 263$ & NI \\
\hline D7S1830 & $7 \mathrm{p} 12$ & 200 & $200 / 212$ & $200 / 212$ & 212 & Biparental \\
\hline D7S1870 & $7 q 11$ & 118 & 118 & 118 & $118 / 120$ & NI \\
\hline D7S634 & $7 q 21$ & $136 / 144$ & $138 / 144$ & $138 / 144$ & $126 / 138$ & Biparental \\
\hline D7S527 & $7 q 21$ & 291 & $291 / 295$ & $291 / 295$ & $273 / 295$ & Biparental \\
\hline D7S1824 & $7 q 34$ & $169 / 173$ & 173 & 173 & 173 & NI \\
\hline D7S550 & $7 q 36$ & $189 / 193$ & 189 & 189 & $187 / 189$ & NI \\
\hline D9S168 & 9 p23 & $228 / 230$ & $228 / 234$ & $228 / 234$ & $234 / 238$ & Biparental \\
\hline D9S171 & $9 \mathrm{p} 21$ & $160 / 164$ & $164 / 166$ & $164 / 166$ & $156 / 166$ & Biparental \\
\hline D10S1580 & $10 \mathrm{p} 12$ & $140 / 148$ & $140 / 150$ & $140 / 150$ & $142 / 150$ & Biparental \\
\hline D10S198 & $10 \mathrm{q} 24$ & $183 / 197$ & $183 / 197$ & $183 / 197$ & 183 & NI \\
\hline D10S1268 & $10 \mathrm{q} 24$ & $137 / 163$ & 137 & 137 & 137 & NI \\
\hline D10S221 & $10 \mathrm{q} 26$ & $92 / 104$ & $102 / 104$ & $102 / 104$ & 102 & Biparental \\
\hline D10S209 & $10 q 26$ & 179 & $179 / 203$ & $179 / 203$ & 203 & Biparental \\
\hline D10S1700 & $10 q 26$ & $125 / 127$ & $125 / 127$ & $125 / 127$ & $123 / 127$ & NI \\
\hline D11S2071 & $11 \mathrm{p} 15$ & $184 / 186$ & $186 / 188$ & $186 / 188$ & $182 / 188$ & Biparental \\
\hline D11S922 & $11 \mathrm{p} 15$ & $87 / 111$ & 87 & 87 & 87 & NI \\
\hline D11S988 & $11 \mathrm{p} 15$ & 113 & $113 / 119$ & $113 / 119$ & $119 / 125$ & Biparental \\
\hline D11S902 & $11 \mathrm{p} 15$ & $138 / 144$ & $138 / 146$ & $138 / 146$ & $146 / 154$ & Biparental \\
\hline D11S904 & $11 \mathrm{p} 14$ & $186 / 198$ & $190 / 198$ & $190 / 198$ & $184 / 190$ & Biparental \\
\hline D14S979 & $14 q 32$ & 197/199 & $199 / 201$ & $199 / 201$ & $193 / 201$ & Biparental \\
\hline D14S985 & $14 q 32$ & $227 / 229$ & $227 / 233$ & $227 / 233$ & $233 / 237$ & Biparental \\
\hline D21S1446 & $21 q 22$ & $206 / 222$ & 206 & 206 & 206 & NI \\
\hline DXYS233 & Xp22, Yp11 & $274 / 280$ & $274 / 278$ & $274 / 278$ & $274 / 278$ & NI \\
\hline
\end{tabular}

NI not informative

The Arabic numbers indicate the PCR product sizes (bp). The primer sequences are available in the GDB Human Genome Database (http://www.gdb.org/)

SRS. Indeed, the methylation pattern of this patient would primarily be consistent with demethylation of the paternally derived H19-DMR in roughly half of cells. Although it might be possible that the H19-DMR hypomethylation took place after the twinning, this notion assumes no causal relationship between the H19-DMR hypomethylation and the twinning.

It may be worth pointing out that the twins were females. In this context, the sex ratio of previously reported five monozygotic twin pairs discordant for SRS is not remarkable with a male to female ratio of $2: 3$, and discordance for hypomethylation of the H19-DMR has been confirmed only in a single female twin pair (Nyhan and Sakati 1977; Samn et al. 1990; Bailey et al. 1995; Sagot et al. 1996; Gicquel et al. 2005). However, discordance for BWS and hypomethylation of the KvDMR1 is predominantly manifested by monozygotic female twins with a male to female ratio of 1:11 (Gaston et al. 2001; Weksberg et al. 2002). Since DNMT1 is also required for the maintenance of X-inactivation (Bestor 2000), this may have reduced the amount of DNMT1 enzyme available for the imprint maintenance of the DMRs, increasing the risk of demethylation of DMRs in the monozygotic female twins.

Hypomethylation of the H19-DMR was identified in leukocytes of this patient, but not in the twin sister. This contrasts the previous finding that H19-DMR in leukocytes is hypomethylated in both of the 11-year-old twin sisters discordant for SRS, whereas H19-DMR in skin fibroblasts is hypomethylated in the affected twin only (Gicquel et al. 2005). Such inconsistency may be due to the difference in placental vascular anatomy. While the present twins had no discernible interconnecting vascular anastomosis in the placenta, the twins described by Gicquel et al. (2005) may have shared fetal circulation, which allowed the transfer and engraftment of blood stem cells with demethylated H19-DMR from the affected twin to the unaffected twin. In this regard, since clinical features are similar between the 
twins of this study and those reported by Gicquel et al. (2005), this implies that epimutations of the non-hematopoietic tissues such as skin fibroblasts are responsible for the development of SRS phenotype, whereas epimutations in leukocytes have no discernible clinical effects. For the present twins, since the $\mathrm{X}$-inactivation pattern in leukocytes was nearly identical between the present twins in the probable absence of the shared fetal circulation, it may be that the $\mathrm{X}$-inactivation pattern was established as a normal event simultaneously with, or just prior to, the demethylation of the H19-DMR as an abnormal event.

In summary, we identified monozygotic female twins discordant for SRS and the H19-DMR hypomethylation. This is reminiscent of hypomethylation of the KvDMR1 in one of the twin with BWS (Gaston et al. 2001; Weksberg et al. 2002). Since the H19-DMR and the KvDMR1 contiguously resides on chromosome $11 \mathrm{p} 15$, there may be a regional property for the occurrence of hypomethylation. Further studies will permit a better clarification of the prevalence of the monozygotic twins discordant for SRS and the mechanisms involved in this condition.

Acknowledgments This work was supported by Grants for Child Health and Development (20C-2) and for Research on Children and Families (H18-005) from the Ministry of Health, Labor, and Welfare, by Grants-in-Aid for Scientific Research (priority areas: 16086215; category B: 19390290) and for Young Scientists (B) (19790752) from the Ministry of Education, Culture, Sports, Science and Technology, and by a Grant from Kawano Masanori Memorial Foundation for Promotion of Pediatrics.

\section{References}

Bailey W, Popovich B, Jones KL (1995) Monozygotic twins discordant for the Russell-Silver syndrome. Am J Med Genet 58:101-105

Bell AC, Felsenfeld G (2000) Methylation of a CTCF-dependent boundary controls imprinted expression of the Igf 2 gene. Nature 405:482-485

Bestor TH (2000) The DNA methyltransferases of mammals. Hum Mol Genet 9:2395-2402

Bliek J, Terhal P, van den Bogaard MJ, Maas S, Hamel B, SaliebBeugelaar G, Simon M, Letteboer T, van der Smagt J, Kroes H, Mannens M (2006) Hypomethylation of the H19 gene causes not only Silver-Russell syndrome (SRS) but also isolated asymmetry or an SRS-like phenotype. Am J Hum Genet 78:604-614

Eggermann T, Schonherr N, Meyer E, Obermann C, Mavany M, Eggermann K, Ranke MB, Wollmann HA (2006) Epigenetic mutations in $11 \mathrm{p} 15$ in Silver-Russell syndrome are restricted to the telomeric imprinting domain. J Med Genet 43:615-616

Gaston V, Le Bouc Y, Soupre V, Burglen L, Donadieu J, Oro H, Audry G, Vazquez MP, Gicquel C (2001) Analysis of the methylation status of the KCNQ1OT and H19 genes in leukocyte
DNA for the diagnosis and prognosis of Beckwith-Wiedemann syndrome. Eur J Hum Genet 9:409-418

Gicquel C, Rossignol S, Cabrol S, Houang M, Steunou V, Barbu V, Danton F, Thibaud N, Le Merrer M, Burglen L, Bertrand AM, Netchine I, Le Bouc Y (2005) Epimutation of the telomeric imprinting center region on chromosome $11 \mathrm{p} 15$ in SilverRussell syndrome. Nat Genet 37:1003-1007

Hao Y, Crenshaw T, Moulton T, Newcomb E, Tycko B (1993) Tumour-suppressor activity of H19 RNA. Nature 365:764-767

Hark AT, Schoenherr CJ, Katz DJ, Ingram RS, Levorse JM, Tilghman SM (2000) CTCF mediates methylation-sensitive enhancerblocking activity at the H19/Igf2 locus. Nature 405:486-489

Hirasawa R, Chiba H, Kaneda M, Tajima S, Li E, Jaenisch R, Sasaki $\mathrm{H}$ (2008) Maternal and zygotic Dnmt1 are necessary and sufficient for the maintenance of DNA methylation imprints during preimplantation development. Genes Dev 22:1607-1616

Hitchins MP, Stanier P, Preece MA, Moore GE (2001) Silver-Russell syndrome: a dissection of the genetic aetiology and candidate chromosomal regions. J Med Genet 38:810-819

Juan V, Crain C, Wilson C (2000) Evidence for evolutionarily conserved secondary structure in the H19 tumor suppressor RNA. Nucleic Acids Res 28:1221-1227

Leighton PA, Ingram RS, Eggenschwiler J, Efstratiadis A, Tilghman SM (1995a) Disruption of imprinting caused by deletion of the H19 gene region in mice. Nature 375:34-39

Leighton PA, Saam JR, Ingram RS, Stewart CL, Tilghman SM (1995b) An enhancer deletion affects both H19 and Igf2 expression. Genes Dev 9:2079-2089

Muroya K, Kosho T, Ogata T, Matsuo M (1999) Female carriers of Xp22.3 deletion including MRX locus. Am J Med Genet 84:384-385

Netchine I, Rossignol S, Dufourg MN, Azzi S, Rousseau A, Perin L, Houang M, Steunou V, Esteva B, Thibaud N, Demay MC, Danton F, Petriczko E, Bertrand AM, Heinrichs C, Carel JC, Loeuille GA, Pinto G, Jacquemont ML, Gicquel C, Cabrol S, Le Bouc Y (2007) 11p15 imprinting center region 1 loss of methylation is a common and specific cause of typical RussellSilver syndrome: clinical scoring system and epigenetic-phenotypic correlations. J Clin Endocrinol Metab 92:3148-3154

Nyhan WL, Sakati NO (1977) Silver syndrome. In: Genetic and malformation syndromes in clinical medicine. Yearbook Medical Publisher, Chicago, pp 298-300

Sagot P, David A, Talmant C, Pascal O, Winer N, Boog G (1996) Russell-Silver syndrome: an explanation for discordant growth in monozygotic twins. Fetal Diagn Ther 11:72-78

Samn M, Lewis K, Blumberg B (1990) Monozygotic twins discordant for the Russell-Silver syndrome. Am J Med Genet 37:543-545

Thorvaldsen JL, Duran KL, Bartolomei MS (1998) Deletion of the H19 differentially methylated domain results in loss of imprinted expression of H19 and Igf2. Genes Dev 12:3693-3702

Vu TH, Li T, Nguyen D, Nguyen BT, Yao XM, Hu JF, Hoffman AR (2000) Symmetric and asymmetric DNA methylation in the human IGF2-H19 imprinted region. Genomics 64:132-143

Weksberg R, Shuman C, Caluseriu O, Smith AC, Fei YL, Nishikawa J, Stockley TL, Best L, Chitayat D, Olney A, Ives E, Schneider A, Bestor TH, Li M, Sadowski P, Squire J (2002) Discordant KCNQ1OT1 imprinting in sets of monozygotic twins discordant for Beckwith-Wiedemann syndrome. Hum Mol Genet 11:1317-1325 